\title{
Pseudoaneurisma de la fibrosa mitro aórtica en endocarditis de aorta bicúspide. A propósito de un caso
}

\author{
Pseudo-aneurism of the mitral aortic intervalvular fibrosa in a patient with bicuspid aortic valve \\ endocarditis. A case report
}

Aida Rotta ${ }^{1, a}$, Roy Dueñas ${ }^{1, a}$, Renzo Rodriguez ${ }^{2, b}$, Carlos Martorell ${ }^{2, b}$.

RESUMEN

El pseudoaneurisma de la fibrosa mitro aórtica es una complicación infrecuente pero potencialmente fatal de la endocarditis infecciosa de válvula aórtica. El diagnóstico de pseudoaneurisma se realiza mediante una técnica de imagen, principalmente la ecocardiografía transtorácica y transesofágica, en la que se evidencia una cavidad perivalvular pulsátil con expansión sistólica y colapso diastólico localizada en la zona mitro-aórtica. Por el riesgo de complicaciones, en la mayoría de pacientes, se recomienda el reemplazo de válvula aórtica con cierre del pseudoaneurisma. Se presenta un caso de pseudoaneurisma de la fibrosa mitroaórtica en un paciente adulto joven con endocarditis de válvula aórtica bicúspide.

PALABRAS CLAVE: Pseudoaneurisma, endocarditis bacteriana, válvula aórtica. (Fuente: DeCS BIREME).

\section{SUMMARY}

Pseudoaneurysm of the mitral-aortic intervalvular fibrosa is an infrequent but potentially fatal complication of aortic valve endocarditis. Diagnostic is done by an imaging technique, especially transthoracic and transesophageal echocardiography, consisting of a pulsatile cavity with systolic expansion and diastolic collapse located in the mitral-aortic intervalvular fibrosa. Due to the risk of complications, aortic valve replacement and closure of the pseudoaneurysm is recommended. We present a case of pseudoaneurysm of the mitral-aortic intervalvular fibrosa in a patient presenting with bicuspid aortic valve endocarditis

KEY WORDS: Pseudoaneurysm, endocarditis, bicuspid aortic valve. (Source: MeSH NLM)

\section{INTRODUCCIÓN}

La endocarditis infecciosa (EI) es una enfermedad infecciosa grave y poco frecuente cuya incidencia anual es de 3 a 7 por 100000 personas-año (1). La valvulopatía reumática, la cardiopatía congénita cianótica no corregida, el uso de dispositivos intracardiacos y válvulas protésicas, el antecedente de endocarditis infecciosa previa y el uso de drogas intravenosas son los factores de riesgo más importantes (2-5). Con menor frecuencia, también se asocia a lesiones valvulares degenerativas, hemodiálisis,

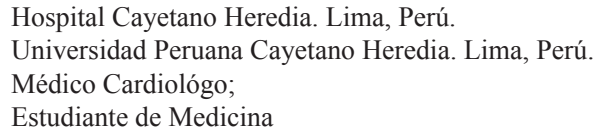


diabetes e infección por VIH. No obstante, hasta 50\% de los casos se puede presentar en pacientes sin estos antecedentes (6).

La extensión local de la endocarditis infecciosa con compromiso del aparato perivalvular o perianular se asocia a desarrollo de insuficiencia cardiaca congestiva y mayor mortalidad. Su frecuencia es variable, afectando entre 10 a $40 \%$ de endocarditis de válvula nativa, especialmente de válvula aórtica (3). La consecuencia de esta complicación es la formación de abscesos, pseudoaneurismas, y fistulas. El absceso perivalvular es una complicación que ocurre con mayor frecuencia en endocarditis de válvula protésica y en infección por Staphylococcus aureus $(7,8)$. La invasión de un absceso al septo membranoso y nodo AV puede ocasionar un bloqueo aurículoventricular. Asimismo, los abscesos no tratados pueden crear tractos fistulosos produciendo shunts causando una mortalidad de $41 \%$ (7). Entre las complicaciones descritas, el pseudoaneurisma se asocia a daño valvular y perivalvular muy severo, requiriendo manejo quirúrgico temprano (4).

El diagnóstico de endocarditis infecciosa ha mejorado significativamente gracias a la ecocardiografía. Un resultado positivo (vegetación valvular, absceso o deshiscencia de válvula protésica) se considera un criterio mayor en los criterios de Duke modificados (1). Adicionalmente, la ecocardiografía brinda información sobre el impacto hemodinámico de la enfermedad y permite la evaluación de la función ventricular (8). La ecocardiografía transesofágica (ETE) tiene mayor sensibilidad y especificidad y está indicada cuando la ecocardiografía transtorácica (ETT) es negativa en un paciente con sospecha de EI, la calidad de imagen es inadecuada con ETT y en pacientes con válvulas protésicas o dispositivos intracardiacos (6). La ETE también permite evaluar con mayor precisión la presencia de abscesos, perforación valvar y pseudoaneurismas; por lo que se puede considerar realizarla aún cuando la ETT fue suficiente para llegar al diagnóstico de EI (8).

A continuación, se presenta el caso de un paciente con endocarditis infecciosa de válvula aórta bicúspide complicada con un pseudoaneurisma de la fibrosa mitro aórtica, entidad rara de alta morbi mortalidad.

\section{PRESENTACIÓN DEL CASO}

Varón de 24 años de edad, natural de Tarapoto, San Martín, procedente de San Juan de Miraflores sin antecedentes de importancia conocidos. Ingresó al servicio de emergencia con un tiempo de enfermedad de un mes caracterizado por sensación de alta térmica, malestar general y baja de peso de $6 \mathrm{~kg}$. Al examen, se encontraba afebril, hemodinámicamente estable; en el examen cardiovascular, se auscultó soplo sistólico IV/ VI en foco aórtico irradiado a cuello y soplo sistólico en foco mitral II/VI.

El electrocardiograma inicial mostró taquicardia sinusal e hipertrofia ventricular izquierda (Cornell positivo). En la ETT inicial se observó una válvula aórtica bicúspide con valvas engrosadas con múltiples imágenes nodulares sugerentes de vegetaciones, estenosis y regurgitación moderada de válvula aórtica, regurgitación mitral leve con función sistólica y motilidad de ventrículo izquierdo conservados. Se evidenció imagen sospechosa de extensión perivalvular (Figura 1).

Los exámenes de laboratorio mostraron anemia leve normocítica normocrómica y PCR elevada. Se le realizaron cuatro tomas de hemocultivo en las que se encontró Streptococcus alfa hemolítico pansensible.

En la ETE se evidenció hipertrofia significativa del ventrículo izquierdo, válvula aórtica bicúspide con valvas engrosadas con múltiples imágenes nodulares compatibles con vegetaciones, la más grande de $6 \mathrm{~mm}$, apertura disminuida, perforación de la valva derecha y prolapso del seno de valsalva correspondiente asociada a regurgitación y estenosis valvular moderada. Se halló una imagen semicircular anecogénica en zona mitroaórtica con paredes ecogénicas, de 2,2 cm de base y $1 \mathrm{~cm}$ de diámetro transversal con pared de $2 \mathrm{~mm}$ con flujo color en su interior. El resto de las válvulas no tenían alteraciones. La conclusión fue endocarditis de

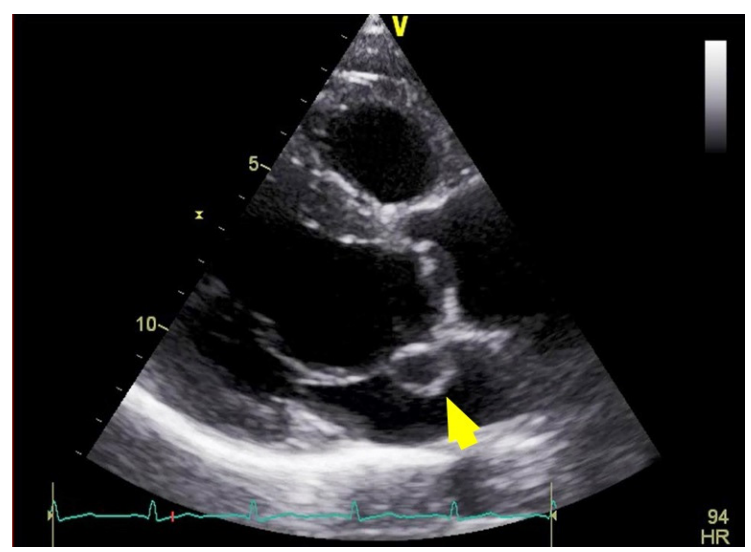

Figura 1. Ecocardiografía transtorácica en la que se evidencia engrosamiento de las válvula aórtica e imagen semicircular pulsátil entre la válvula aórtica y mitral. 
válvula aórtica bicúspide con estenosis e insuficiencia moderadas complicada con pseudoaneurisma de la unión mitroaórtica, hipertrofia significativa de ventrículo izquierdo con función sistólica y motilidad de ventrículo izquierdo conservadas (Figura 2).

Durante su estadía en el servicio de Medicina, paciente presentó cuadro de amaurosis en ojo izquierdo secundario a oclusión de la arteria central de retina izquierda, y posteriormente un émbolo arterial a miembro inferior izquierdo.

El paciente fue sometido a cirugía para recambio de válvula aórtica con prótesis mecánica, 5 días después en su día 28 de hospitalización. Se evidenció engrosamiento valvar por endocarditis y absceso subaortico de $2,5 \mathrm{~cm}$ que perforaba el septum interventricular. Se colocó parche pericárdico en septum interventricular. La anatomía patológica mostró tejido fibrovascularizado, tapizado por material fibrinohemático y purulento con presencia de células gigantes multinucleadas tipo cuerpo extraño y calcificaciones aisladas (Figura 3).

En su evolución post operatoria, presentó convulsiones generalizadas tónico clónicas. Se realizó TAC cerebral en la que se reportó una imagen hipodensa en región occipital derecha en relación a lesión isquémica con edema citotóxico de etiología cardioembólica. Posteriormente, presentó secuelas motoras y persiste disminución de agudeza visual de ojo izquierdo por atrofia de nervio óptico. Además,

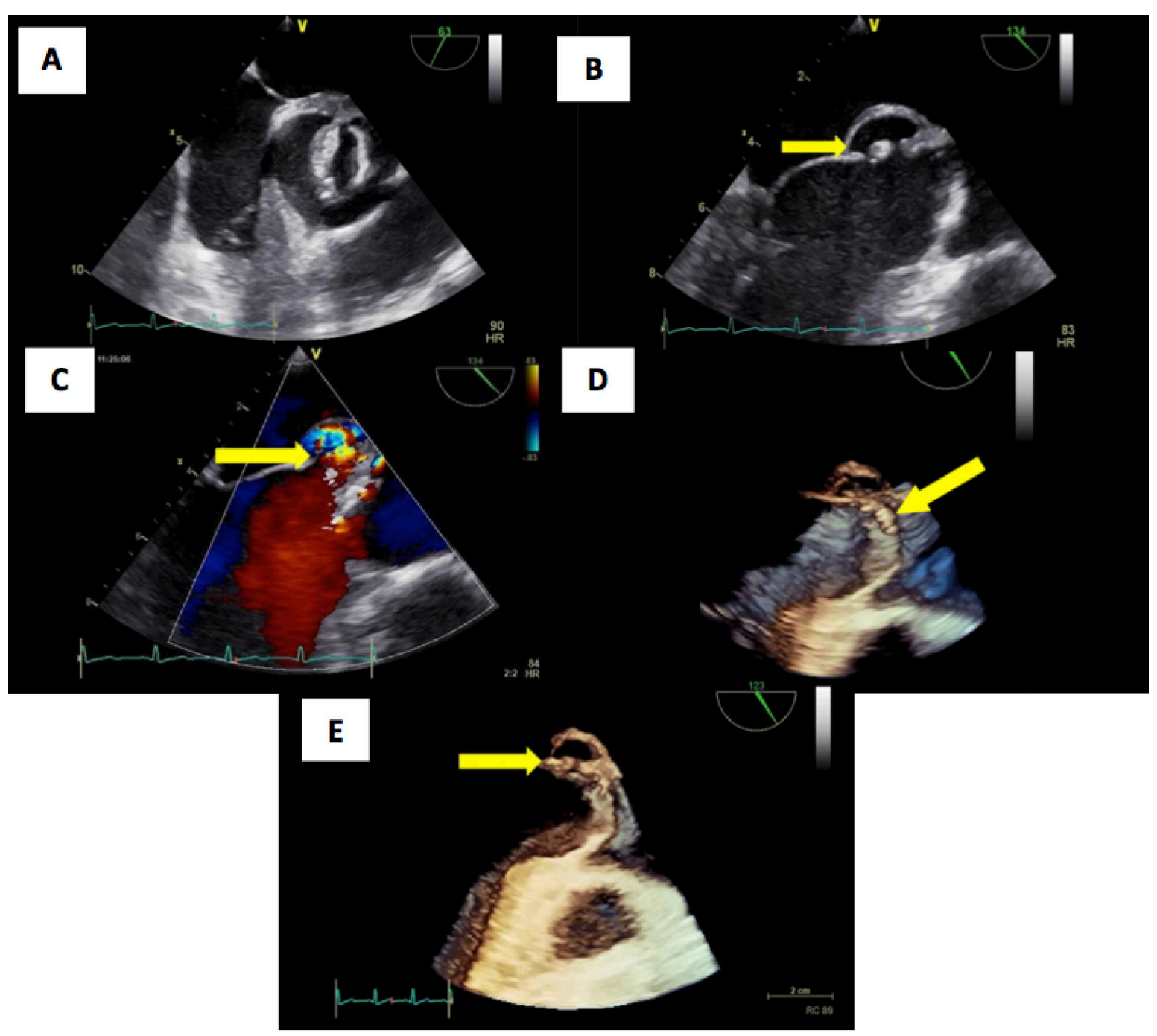

Figura 2. Ecocardiografía tranesofágica A:Válvula aórtica bicúspide B: Imagen de pseudoaneurisma en la región mitroaórtica. C: Flujo hacia el interior del pseudoaneurisma con Doppler color D: Vision en 3D que muestra engrosamiento y presencia de nódulos en la válvula aórtica. E: Reconstrucción en 3D del pseudoaneurisma. 


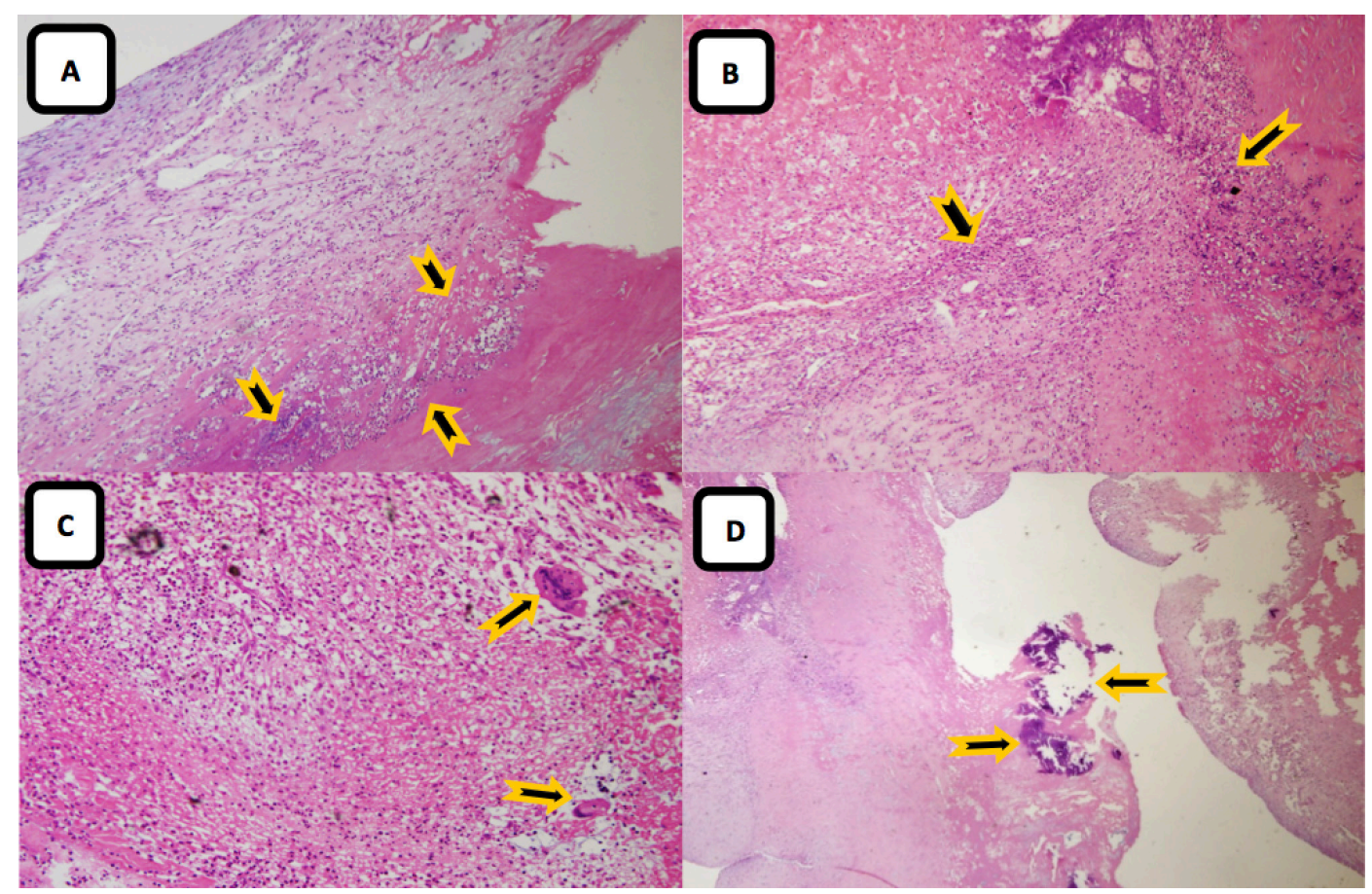

Figura 3. A y B: Tejido fibrovascularizado, tapizado por material fibrinohemático y purulento con macrófagos y neutrófilos (100X). C: Células gigantes multinucleadas tipo cuerpo extraño (400X) y D: calcificaciones aisladas (40X).

presentó síndrome post pericardiotomía tratado exitosamente con colchicina. Fue dado de alta con tratamiento anticoagulante y anticonvulsivante en su día $\mathrm{PO}_{26}$.

\section{DISCUSIÓN}

En la endocarditis infecciosa de válvula aórtica, la extensión perivalvular ocurre con mayor frecuencia en la región fibrosa intervalvular mitral-aórtica (5). Esta estructura está localizada en la zona interanular, entre la valva aórtica coronariana izquierda, la valva no coronariana y la valva anterior de la válvula mitral, adyacente al tracto de salida del ventrículo izquierdo $(5,9) . \mathrm{Su}$ característica fibrosa y relativamente avascular la torna propensa a la infección y a lesiones mecánicas.

El pseudonaneurisma de la fibrosa mitroaórtica tiene como principales etiologías la endocarditis infecciosa y la cirugía de la válvula aórtica; y como principales factores contributorios la regurgitación de válvula aórtica y la vávula aórtica bicúspide como en este caso (5). Otras posibles causas son el trauma torácico y el origen congénito. La presentación clínica es heterogénea. En una revisión de 89 casos realizada por Sudhakar et al (5), la mayoría de pacientes (39\%) presentó síntomas y signos de endocarditis infecciosa activa; la disnea e insuficiencia cardiaca le siguieron en frecuencia (16\%). Las complicaciones embolicas se presentaron en $12 \%$ de los casos; y el dolor torácico, en $10 \%$. Hasta $9 \%$ fueron asintomáticos $(5,9)$. Las complicaciones del pseudoaneurisma son la formación de fístulas, compresión de la arteria coronaria, destrucción valvular o ruptura del mismo hacia el espacio pericárdico (5).

El diagnóstico de pseudoaneurisma se realiza mediante una técnica de imagen. En el ecocardiograma se evidencia una cavidad perivalvular pulsátil con expansión sistólica y colapso diastólico localizada en la region de la fibrosa mitroaórtica $(5,8,9)$. Con el uso del Doppler se puede observar flujo en su interior (8).

El pseudoaneurisma es manifiestación de infección local no controlada y una indicación para manejo quirúgico de urgencia (Clase I, Nivel de evidencia B) (4). Las complicaciones embólicas en este paciente ocurrieron probablemente debido a la demora en el manejo quirúrgico definitivo. El paciente no fue operado de manera oportuna a causa de la falta de materiales quirúrgicos. El reemplazo de válvula aórtica se recomienda en la mayoría de pacientes con endocarditis infecciosa que presenten 
un pseudoaneurisma que se localice adyacente al anillo aórtico (5). Cuando se sospecha de una causa mecánica y no hay evidencia de infección, se podría realizar solo el cierre del aneurisma utilizando un graft sintético o pericárdico (10). También se ha propuesto la posibilidad de cierre percutáneo (11). Por otra parte, se ha reportado casos de manejo conservador en pacientes con pseudonaneurisma pequeño no complicado, en los que los pacientes permanecieron asintomáticos luego de años de seguimiento pese al aumento gradual del tamaño de la lesión (12).

\section{Agradecimientos:}

Al Dr. Jaime Cáceres Pizarro y Dra. Jenny Sianca, por la preparación y descripción de las láminas anatomopatológicas.

\section{Declaración de financiamiento y conflictos de intereses:}

El reporte fue financiado por los autores. Los autores declaran no tener conflicto de intereses.

\section{Contribución de autoría:}

AR, RD, RR, CM: participaron en la concepción, elaboración, análisis y redacción del reporte de caso.

\section{Correspondencia}

Carlos Martorell Moreau

Calle Las Palomas 380. Surquillo Lima, Perú.

Correo electronico: carlos.martorell.m@upch.pe

Teléfono: 51940402586.

\section{REFERENCIAS BIBLIOGRÁFICAS}

1. Baddour LM, Wilson WR, Bayer AS, et al. Infective Endocarditis in Adults: Diagnosis, Antimicrobial Therapy, and Management of Complications: A Scientific Statement for Healthcare Professionals From the American Heart Association. Circulation. 2015; 132(15):1435-1486.
2. Fernández-Hidalgo N, Almirante B. La endocarditis infecciosa en el siglo xxi: cambios epidemiológicos, terapéuticos y pronósticos Enferm Infecc Microbiol Clin. 2012; 30(7):394-406.

3. Anguera I, Miro JM, Evangelista A, et al. Periannular complications in infective endocarditis involving native aortic valves. Am J Cardiol. 2006; 98:12541260.

4. Habid G. 2015 ESC Guidelines for the management of infective endocarditis: The Task Force for the Management of Infective Endocarditis of the European Society of Cardiology (ESC). Endorsed by: European Association for Cardio-Thoracic Surgery (EACTS), the European Association of Nuclear Medicine (EANM). Eur Heart J. 2015; 36(44):3075-3128.

5. Sudhakar S1, Sewani A, Agrawal M, Uretsky BF. Pseudoaneurysm of the mitral-aortic intervalvular fibrosa (MAIVF): A comprehensive review. J Am Soc Echocardiogr. 2010; 23(10):1009-1018.

6. Hoen B, Duval X. Infective Endocarditis. N Engl J Med. 2013; 368(15):1425-33.

7. Prendergast BD, Tornos P. Surgery for infective endocarditis. Circulation. 2010; 121(9):1141-1152.

8. Cahill TJ, Prendergast BD. Infective endocarditis. The Lancet. 2016; 387(10021):882-893.

9. Xie M, Li Y, Cheng TO, Wang X, Lu Q, He L, et al. Pseudoaneurysm of the mitral-aortic intervalvular fibrosa. Int J Cardiol. 2013; 166(1):2-7.

10. Spampinato RA, Borger MA, Strotdrees E, Mohr FW. Pseudoaneurysm of the mitral-aortic intervalvular fibrosa as a complication after minimally invasive mitral valve repair. Interact Cardiovasc Thorac Surg. 2013; 16(3):396-398.

11. Jiménez Valero S, García E, González Pinto Á, Delcán JL. Cierre percutáneo de seudoaneurisma de la fibrosa mitroaórtica. Rev Esp Cardiol. 2005; 58(12):14731475.

12. Chieppa R, Scardigno, Deluca G, Capodivento S, Quinto N, Cicala M. Conservative treatment of a pseudoaneurysm of the mitral-aortic intervalvular fibrosa: 9 years of follow-up. G Ital Cardiol (Rome). 2017 Jun; 18(6):525-528.

Recibido: 10/02/2018

Aceptado: 03/06/2018 